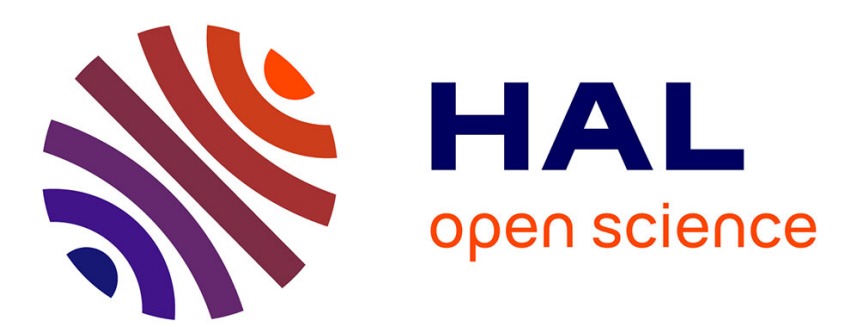

\title{
Riccati nonlinear observer for velocity-aided attitude estimation of accelerated vehicles using coupled velocity measurements
}

\author{
Minh-Duc Hua, Tarek Hamel, Claude Samson
}

\section{- To cite this version:}

Minh-Duc Hua, Tarek Hamel, Claude Samson. Riccati nonlinear observer for velocity-aided attitude estimation of accelerated vehicles using coupled velocity measurements. CDC 2017 56th IEEE Conference on Decision and Control, Dec 2017, Melbourne, Australia. pp.2428-2433, 10.1109/CDC.2017.8264005 . hal-01628178

\section{HAL Id: hal-01628178 \\ https://hal.science/hal-01628178}

Submitted on 2 Nov 2017

HAL is a multi-disciplinary open access archive for the deposit and dissemination of scientific research documents, whether they are published or not. The documents may come from teaching and research institutions in France or abroad, or from public or private research centers.
L'archive ouverte pluridisciplinaire HAL, est destinée au dépôt et à la diffusion de documents scientifiques de niveau recherche, publiés ou non, émanant des établissements d'enseignement et de recherche français ou étrangers, des laboratoires publics ou privés. 


\title{
Riccati nonlinear observer for velocity-aided attitude estimation of accelerated vehicles using coupled velocity measurements
}

\author{
Minh-Duc Hua, Tarek Hamel, Claude Samson
}

\begin{abstract}
Motivated by drone autonomous navigation applications we address a novel problem of velocity-aided attitude estimation by combining two linear velocity components measured in a body-fixed frame and a linear velocity component measured in an inertial frame with the measurements of an Inertial Measurement Unit (IMU). The main contributions of the present paper are the design of a Riccati nonlinear observer, which may be viewed as deterministic versions of an Extended Kalman filter (EKF), and an analysis of observability conditions under which local exponential stability of the observer is achieved. Reported simulation results further indicate that the observer's domain of convergence is large.
\end{abstract}

\section{INTRODUCTION}

The development of reliable attitude (i.e. orientation) estimators is a key requirement for efficient automatic control of drones. Most existing attitude observers exploit the measurements of an IMU under the assumption of weak accelerations of the vehicle to justify the direct use of accelerometer measurements for the estimation of the gravity direction in a body-fixed frame [2], [7], [10], [13]. The violation of this assumption, when the vehicle undergoes sustained accelerations, jeopardizes the accuracy of the attitude estimate (cf. [6]). To overcome this problem velocity-aided attitude observers have been developed in the literature by fusing IMU measurements with the vehicle's linear velocity measurements done either in an inertial frame [4], [6], [9], [12], [15] or in a body-fixed frame [1], [3], [8], [17]. The present paper addresses a new problem of velocity-aided attitude estimation where the vehicle's linear velocity is measured partly in a body-fixed frame and partly in an inertial frame. A motivating application of this work is related to quadrotor UAV navigation in situations where linear velocity's components along two body axes orthogonal to the thrust direction and expressed in a body-fixed frame can be derived from accelerometer measurements combined with an aerodynamic linear drag model [11], [14] and where the linear velocity's vertical component expressed in an inertial frame can be obtained from barometer measurements. The important nonlinearities resulting from the use of such measurements render the design of an attitude observer significantly more complex than when all the linear velocity's components are measured in a single frame, either inertial or body-fixed. They also exclude the possibility of proving semi-global, or almost-global, stability results similar to

M.-D. Hua and T. Hamel are with I3S, Université Côte d'Azur, CNRS, Sophia Antipolis, France, hua(thamel)@i3s.unice.fr.

C. Samson is with INRIA and I3S UCA-CNRS, Sophia Antipolis, France, claude.samson@inria.fr,csamson@i3s.unice.fr. these derived in [6] and [8] in the simpler case of complete linear velocity measurements in a single frame.

The design of the observer proposed in this paper is adapted from a recent deterministic Riccati observer design framework [5] that relies on the solutions to the Continuous Riccati Equation (CRE) and encompasses EKF solutions. Accordingly, good conditioning of the solutions to the CRE and, subsequently, exponential stability of the obtained observer rely on conditions of uniform observability whose satisfaction calls for a specific analysis. Since only local stability is demonstrated simulation results are useful to get complementary indications about the performance and the size of the basin of attraction of the proposed observer.

The paper is organized as follows. Notation, system equations, and the measurements involved in the observer design are specified in Section II. In the same section some basic definitions and conditions about system observability are recalled, together with elements of the deterministic Riccati observer design framework proposed in [5]. In Section III the observer expressions are specified, and an analysis of associated observability conditions is carried out in Section IV. Simulation results illustrating the performance of the observer and showing that its domain of convergence can be large are reported in Section V. Conclusions then follow.

\section{Preliminary material}

\section{A. Notation}

- $\left\{e_{1}, e_{2}, e_{3}\right\}$ denotes the canonical basis of $\mathbb{R}^{3}$ and $[\cdot]_{\times}$ denotes the skew-symmetric matrix associated with the cross product, i.e., $[u]_{\times} v=u \times v, \forall u, v \in \mathbb{R}^{3}$. The identity matrix of $\mathbb{R}^{n \times n}$ is denoted as $I_{n}$ and $\pi_{x} \triangleq I_{3}-x x^{\top}, \forall x \in S^{2}$ (the unit 2-sphere), is the projection operator onto the plane orthogonal to $x$. Note that $\pi_{x}=-[x]_{\times}^{2}, \forall x \in S^{2}$.

- $\{\mathcal{I}\}=\left\{O ; \vec{\imath}_{0}, \vec{\jmath}_{0}, \vec{k}_{0}\right\}$ denotes an inertial frame attached to the earth, typically chosen as the north-east-down frame, and $\{\mathcal{B}\}=\{G ; \vec{\imath}, \vec{\jmath}, \vec{k}\}$ is a body-fixed frame whose origin is the vehicle's center of mass $G$.

- The vehicle's attitude is represented by a rotation matrix $R \in \mathrm{SO}(3)$ of the frame $\{\mathcal{B}\}$ relative to $\{\mathcal{I}\}$. The element at the intersection of the $i^{\text {th }}$ row and $j^{\text {th }}$ column of $R$ is denoted as $R_{i, j}$, with $i, j \in\{1,2,3\}$.

- $V \in \mathbb{R}^{3}$ and $\Omega \in \mathbb{R}^{3}$ are the vectors of coordinates of the vehicle's linear and angular velocities expressed in $\{\mathcal{B}\}$. The linear velocity expressed in $\{\mathcal{I}\}$ is denoted as $v \in \mathbb{R}^{3}$ so that $v=R V$.

\section{B. System equations and measurements}

The vehicle's attitude satisfies the differential equation 


$$
\dot{R}=R[\Omega]_{\times}
$$

We assume that the vehicle is equipped with an IMU consisting of a 3-axis gyrometer that measures the angular velocity $\Omega \in \mathbb{R}^{3}$ and of a 3 -axis accelerometer that measures the specific acceleration $a_{\mathcal{B}} \in \mathbb{R}^{3}$, expressed in $\{\mathcal{B}\}$. Using the flat non-rotating Earth assumption, we have [3]

$$
\dot{V}=-[\Omega]_{\times} V+a_{\mathcal{B}}+g R^{\top} e_{3}
$$

with $g$ the gravity constant. A 3-axis magnetometer is also available to measure of the normalized Earth's magnetic field vector expressed in $\{\mathcal{B}\}$. Let $m_{\mathcal{I}}=\left[m_{1}, m_{2}, m_{3}\right]^{\top} \in S^{2}$ denote the known normalised Earth's magnetic field vector expressed in $\{\mathcal{I}\}$. The vectors $m_{\mathcal{I}}$ and $e_{3}$ are usually assumed to be non-collinear so that $R$ can be estimated from the observation (measurements) in the body-fixed frame of the gravity vector and of the Earth's magnetic field vector. The magnetometer thus measures $m_{\mathcal{B}}=R^{\top} m_{\mathcal{I}}$. We further assume that the vehicle is equipped with sensory devices that provide measurements of the two first components of $V$ and the third component of $v$, i.e., $V_{1}, V_{2}$ and $v_{3}$. A possible combination of sensors providing such measurements in the case of a flying drone was evoked in the introduction.

\section{Recalls of observability definitions and conditions}

The following definitions and conditions are classical and just recalled here for the sake of completeness. Consider a linear time-varying (LTV) system

$$
\left\{\begin{array}{l}
\dot{x}=A(t) x+B(t) u \\
y=C(t) x
\end{array}\right.
$$

with $x \in \mathbb{R}^{n}$ the system state vector, $u \in \mathbb{R}^{s}$ the system input vector, and $y \in \mathbb{R}^{m}$ the system output vector.

Definition 1 (uniform observability) System (3) is uniformly observable if there exist $\delta, \mu>0$ such that (s.t.) $\forall t \geq 0$

$$
W(t, t+\delta) \triangleq \frac{1}{\delta} \int_{t}^{t+\delta} \Phi^{\top}(t, s) C^{\top}(s) C(s) \Phi(t, s) d s
$$

with $\Phi(t, s)$ the transition matrix associated with $A(t)$, i.e. such that $\frac{d}{d t} \Phi(t, s)=A(t) \Phi(t, s)$ with $\Phi(t, t)=I_{n}$.

$W(t, t+\delta)$ is called the observability Gramian of System (3). When (4) is satisfied one also says that the pair $(A(t), C(t))$ is uniformly observable. The following useful condition points out a sufficient condition for uniform observability.

Lemma 1 (see [16]) If there exists a matrix-valued function $M(\cdot)$ of dimension $(p \times n)(p \geq 1)$ composed of row vectors of $N_{0}=C, N_{k}=N_{k-1} A+\dot{N}_{k-1}, k=1, \cdots$ such that for some positive numbers $\bar{\delta}, \bar{\mu}$ and $\forall t \geq 0$

$$
\frac{1}{\bar{\delta}} \int_{t}^{t+\bar{\delta}} M^{\top}(s) M(s) d s \geq \bar{\mu} I_{n}
$$

then the observability Gramian of System (3) satisfies condition (4).

\section{Recalls of a Riccati observer design framework}

The proposed observer design is adapted from the deterministic observer design framework reported in [5]. Consider the nonlinear system

$$
\left\{\begin{array}{l}
\dot{x}=A\left(x_{1}, t\right) x+u \\
y=C(x, t) x
\end{array}\right.
$$

with $x=\left[x_{1}^{\top}, x_{2}^{\top}\right]^{\top}, x_{1} \in \mathcal{B}_{r}^{n}$ (the closed ball in $\mathbb{R}^{n}$ of radius $r$ ), $x_{2} \in \mathbb{R}^{n}, y \in \mathbb{R}^{m}, A\left(x_{1}, t\right)$ a continuous matrixvalued function uniformly bounded w.r.t. $t$ and uniformly continuous w.r.t. $x_{1}$ of the form

$$
A\left(x_{1}, t\right)=\left[\begin{array}{cc}
A_{1,1}(t) & 0 \\
A_{2,1}\left(x_{1}, t\right) & A_{2,2}(t)
\end{array}\right]
$$

and $C(x, t)$ a continuous matrix-valued function uniformly bounded w.r.t. $t$ and uniformly continuous w.r.t. $x$. Apply

$$
u=-P C^{\top} Q y
$$

with $P \in \mathbb{R}^{2 n \times 2 n}$ a symmetric positive definite matrix solution to the following CRE

$$
\dot{P}=A P+P A^{\top}-P C^{\top} Q(t) C P+S(t)
$$

with $P(0) \in \mathbb{R}^{2 n \times 2 n}$ a symmetric positive definite matrix, $Q(t) \in \mathbb{R}^{m \times m}$ bounded continuous symmetric positive semidefinite, and $S(t) \in \mathbb{R}^{2 n \times 2 n}$ bounded continuous symmetric positive definite. Then, from Theorem 3.1 and Corollary 3.2 in [5], $x=0$ is locally exponentially stable when $Q(t)$ and $S(t)$ are both larger than some positive matrix and the pair $\left(A^{\star}(t), C^{\star}(t)\right)$, with

$$
A^{\star}(t) \triangleq A(0, t), C^{\star}(t) \triangleq C(0, t)
$$

is uniformly observable.

\section{OBSERVER DESIGN}

Let $\hat{R} \in \mathrm{SO}(3)$ and $\hat{V} \in \mathbb{R}^{3}$ denote the estimates of $R$ and $V$, respectively. The proposed observer is of the form

$$
\left\{\begin{array}{l}
\dot{\hat{R}}=\hat{R}[\Omega]_{\times}-\left[\sigma_{R}\right]_{\times} \hat{R} \\
\dot{\hat{V}}=-[\Omega]_{\times} \hat{V}+a_{\mathcal{B}}+g \hat{R}^{\top} e_{3}-\sigma_{V}
\end{array}\right.
$$

where $\sigma_{R}, \sigma_{V} \in \mathbb{R}^{3}$ are innovation terms specified thereafter. Defining the observer errors

$$
\tilde{R} \triangleq R \hat{R}^{\top}, \tilde{V} \triangleq V-\hat{V}
$$

then the design objective is the exponential stability of $(\tilde{R}, \tilde{V})=\left(I_{3}, 0\right)$. From (1), (2) and (10) one verifies that

$$
\begin{cases}\dot{\tilde{R}} & =\tilde{R}\left[\sigma_{R}\right]_{\times} \\ \dot{\tilde{V}} & =-[\Omega]_{\times} \tilde{V}+g \hat{R}^{\top}\left(\tilde{R}^{\top}-I_{3}\right) e_{3}+\sigma_{V}\end{cases}
$$

The next step consists in working out first order approximations of the error system (11) complemented with first order approximations of the measurement equations. The application to these approximations of the Riccati observer design framework reported in [5] will then provide us with the expressions of the innovation terms $\sigma_{R}$ and $\sigma_{V}$.

For this application we need to make the following technical (but non-restrictive) assumption.

Assumption 1 The vehicle's horizontal velocity $v_{1,2}(t) \triangleq\left[v_{1}(t), v_{2}(t)\right]^{\top}$, horizontal acceleration $\dot{v}_{1,2}(t) \triangleq\left[\dot{v}_{1}(t), \dot{v}_{2}(t)\right]^{\top}$ and angular velocity $\Omega(t)$ are bounded in norm by some non-negative numbers $v_{1,2 \max }$, $\dot{v}_{1,2 \max }$ and $\Omega_{\max }$, i.e. $\left|v_{1,2}(t)\right| \leq v_{\max },\left|\dot{v}_{1,2}(t)\right| \leq \dot{v}_{1,2 \max }$ and $|\Omega(t)| \leq \Omega_{\max }$.

First order approximations of the attitude error equation is derived by considering (local) minimal parametrizations of the three-dimensional group of rotations $\mathrm{SO}(3)$. The parametrization here chosen is the vector part $\tilde{q}$ of the Rodrigues unit quaternion $\tilde{Q}=\left(\tilde{q}_{0}, \tilde{q}\right)$ associated with $\tilde{R}$. Rodrigues formula relating $\tilde{Q}$ to $R$ is 
$\tilde{R}=I_{3}+2[\tilde{q}]_{\times}\left(\tilde{q}_{0} I_{3}+[\tilde{q}]_{\times}\right)=I_{3}+[\tilde{\lambda}]_{\times}+O\left(|\tilde{\lambda}|^{2}\right)$

with $\tilde{\lambda} \triangleq 2 \operatorname{sign}\left(\tilde{q}_{0}\right) \tilde{q}$. Then, in view of the dynamics of $\tilde{R}$ in (11) one verifies (see also [5]) that

$$
\dot{\tilde{\lambda}}=\sigma_{R}+O\left(|\tilde{\lambda}|\left|\sigma_{R}\right|\right)
$$

As for the dynamics of $\tilde{V}$ one obtains

$$
\dot{\tilde{V}}=-[\Omega]_{\times} \tilde{V}+g \hat{R}^{\top}\left[e_{3}\right]_{\times} \tilde{\lambda}+\sigma_{V}+O\left(|\tilde{\lambda}|^{2}\right)
$$

Concerning the measurement of $v_{3}$ one has

$$
\begin{aligned}
& v_{3}-e_{3}^{\top} \hat{R} \hat{V}=e_{3}^{\top}\left(\tilde{R}-I_{3}\right) \hat{R}(\hat{V}+\tilde{V})+e_{3}^{\top} \hat{R} \tilde{V} \\
& \quad=-e_{3}^{\top}[\hat{R} \hat{V}]_{\times} \tilde{\lambda}+e_{3}^{\top} \hat{R} \tilde{V}+O(|\tilde{\lambda}||\tilde{V}|)+O\left(|V||\tilde{\lambda}|^{2}\right)
\end{aligned}
$$

As for the measurement of $m_{\mathcal{B}}$ one obtains

$$
\hat{R} m_{\mathcal{B}} \times m_{\mathcal{I}}=\tilde{R}^{\top} m_{\mathcal{I}} \times m_{\mathcal{I}}=\pi_{m_{\mathcal{I}}} \tilde{\lambda}+O\left(|\tilde{\lambda}|^{2}\right)
$$

In view of relations (12), (13), (14) and (15), by setting the system output vector equal to

$$
y=\left[\begin{array}{c}
V_{1}-\hat{V}_{1} \\
V_{2}-\hat{V}_{2} \\
v_{3}-e_{3}^{\top} \hat{R} \hat{V} \\
\left(\hat{R} m_{\mathcal{B}}\right) \times m_{\mathcal{I}}
\end{array}\right]
$$

one obtains LTV first order approximations in the form (6) with

$$
\left\{\begin{array}{l}
x=\left[\begin{array}{c}
\tilde{\lambda} \\
\tilde{V}
\end{array}\right], x_{1}=\tilde{\lambda}, x_{2}=\tilde{V}, u=\left[\begin{array}{c}
\sigma_{R} \\
\sigma_{V}
\end{array}\right] \\
A=\left[\begin{array}{cc}
0_{3 \times 3} & 0_{3 \times 3} \\
g \hat{R}^{\top}\left[e_{3}\right]_{\times} & -[\Omega]_{\times}
\end{array}\right], C=\left[\begin{array}{cc}
0_{1 \times 3} & e_{1}^{\top} \\
0_{1 \times 3} & e_{2}^{\top} \\
-e_{3}^{\top}[\hat{R} \hat{V}]_{\times} & e_{3}^{\top} \hat{R} \\
\pi_{m_{\mathcal{I}}} & 0_{3 \times 3}
\end{array}\right](16)
\end{array}\right.
$$

From there the proposed observer is given by (10) with $\sigma_{R}$ and $\sigma_{V}$ determined from the input $u$ calculated according to (7) and (8).

\section{OBSERVABiLITy ANALYSis}

According to [5, Corollary 3.2], good conditioning of the solutions $P(t)$ to the CREs and exponential stability of the previously derived observer rely on the uniform observability of the pair $\left(A^{\star}(t), C^{\star}(t)\right)$ obtained by setting $x=0$ in the expressions of the matrices $A$ and $C$ derived previously. In view of (16) one has

$A^{\star}=\left[\begin{array}{cc}0_{3 \times 3} & 0_{3 \times 3} \\ g R^{\top}\left[e_{3}\right]_{\times} & -[\Omega]_{\times}\end{array}\right], C^{\star}=\left[\begin{array}{cc}0_{2 \times 3} \\ -e_{3}^{\top}[v]_{\times} \\ \pi_{m_{\mathcal{I}}} & 0_{3 \times 3}\end{array}\right]$

with $\Delta \triangleq\left[e_{1}\left|e_{2}\right| R^{\top} e_{3}\right]^{\top} \in \mathbb{R}^{3 \times 3}$.

Define $D=\left[\begin{array}{ll}D_{1,1} & D_{1,2} \\ D_{2,1} & D_{2,2}\end{array}\right] \triangleq M^{\top} M$, with $M \triangleq\left[\begin{array}{cc}C^{\star} \\ C^{\star} A^{\star}+\dot{C}^{\star}\end{array}\right]$. From Lemma 1 the pair $\left(A^{\star}, C^{\star}\right)$ is uniformly observable if $\exists \delta>0, \mu>0$ such that

$$
\frac{1}{\delta} \int_{t}^{t+\delta} D(s) d s \geq \mu I_{6}, \forall t>0
$$

Straightforward calculations yield

$$
\left\{\begin{aligned}
D_{1,1}= & \pi_{m_{\mathcal{I}}}+g^{2} \pi_{e_{3}}-g^{2}\left(e_{3} \times R e_{3}\right)\left(e_{3} \times R e_{3}\right)^{\top} \\
& +\left(e_{3} \times v\right)\left(e_{3} \times v\right)^{\top}+\left(e_{3} \times \dot{v}\right)\left(e_{3} \times \dot{v}\right)^{\top} \\
D_{1,2}= & D_{2,1}^{\top}=[v]_{\times} e_{3} e_{3}^{\top} R+g\left[e_{3}\right]_{\times} R \pi_{e_{3}}[\Omega]_{\times} \\
D_{2,2}= & \Delta^{\top} \Delta-[\Omega]_{\times} \pi_{e_{3}}[\Omega]_{\times}
\end{aligned}\right.
$$

The following proposition points out more explicit conditions whose satisfaction ensures uniform observability and thus local exponential stability of the proposed observer. Its proof is based on the fact that (18) is equivalent to

$$
\frac{1}{\delta} \int_{t}^{t+\delta} X^{\top} D(s) X d s \geq \mu|X|^{2}
$$

$\forall X=\left[x^{\top}, y^{\top}\right]^{\top} \in \mathbb{R}^{6}$ and $\forall t \geq 0$, which, by simple computations, is also equivalent to $\forall x, y \in \mathbb{R}^{3}$ :

$$
\begin{aligned}
& \frac{1}{\delta} \int_{t}^{t+\delta}\left(\left|m_{\mathcal{I}} \times x\right|^{2}+g^{2}\left|\left[R(s) e_{3}\right]_{\times}\left[e_{3}\right]_{\times} x\right|^{2}\right. \\
& \quad+\left(\left(e_{3} \times v(s)\right)^{\top} x\right)^{2}+\left(\left(e_{3} \times \dot{v}(s)\right)^{\top} x\right)^{2} \\
& \quad+y_{1}^{2}+y_{2}^{2}+\left(\left(R^{\top}(s) e_{3}\right)^{\top} y\right)^{2}+\left|\left[e_{3}\right]_{\times}[\Omega(s)]_{\times} y\right|^{2} \\
& \quad+2 g\left(R^{\top}(s)\left[R(s) e_{3}\right]_{\times}\left[e_{3}\right]_{\times} x\right)^{\top}\left(\left[e_{3}\right]_{\times}[\Omega(s)]_{\times} y\right) \\
& \left.\quad+2\left(\left(v(s) \times e_{3}\right)^{\top} x\right)\left(\left(R^{\top}(s) e_{3}\right)^{\top} y\right)\right) d s \\
& \geq \mu\left(|x|^{2}+|y|^{2}\right)
\end{aligned}
$$

Proposition 1 (Proof in Appendix A) Assume that

$$
\exists \delta, \rho>0 \text { s.t. } \frac{1}{\delta} \int_{t}^{t+\delta} R_{3,3}^{2}(s) d s \geq \rho, \forall t \geq 0
$$

Then for the following cases.

1) Motion along the vertical direction, i.e. $v(t) \times e_{3} \equiv 0$;

2) Pure translation, i.e. $\Omega(t) \equiv 0$;

3) Motion such that $v_{1,2 \max } \Omega_{\max } \leq \frac{g \rho}{\sqrt{6}}$, with $v_{1,2 \max }$ and $\Omega_{\max }$ standing for the bounds of $v_{1,2}$ and $\Omega$ defined in Assumption 1;

4) Persistently accelerated translational motion such that $\exists \rho_{1}>0, \forall t \geq 0:$

$$
\frac{1}{\delta} \int_{t}^{t+\delta} \dot{v}_{1,2}(s) \dot{v}_{1,2}^{\top}(s) d s \geq \rho_{1} I_{2}
$$

5) Persistently accelerated translational motion such that $\exists \rho_{2}>0, \forall t \geq 0$ :

$$
\frac{1}{\delta} \int_{t}^{t}\left(m_{2} \dot{v}_{1}(s)-m_{1} \dot{v}_{2}(s)\right)^{2} d s \geq \rho_{2}
$$

condition (18) is satisfied. Consequently, the pair $\left(A^{\star}, C^{\star}\right)$ given by (17) is uniformly observable and the equilibrium $(\tilde{R}, \tilde{V})=\left(I_{3}, 0\right)$ of the proposed Riccati observer is locally exponentially stable.

Some comments on Proposition 1 are provided next.

- The third case of Proposition 1 encompasses the first and second cases.

- Condition (21) is violated when the gravity direction expressed in the body-fixed frame $\{\mathcal{B}\}$ (i.e. $R^{\top} e_{3}$ ) always remains on the plane spanned by $e_{1}$ and $e_{2}$ or approaches it asymptotically. For instance, if $\forall t: R(t)^{\top} e_{3} \in \operatorname{span}\left(e_{1}, e_{2}\right)$ then the uniform observability sufficient condition (18) is not satisfied since in that case the last row and last column of $D$ (given by (19)) are equal to zero. However, this very particular situation of non observability is not supposed to occur in the case of VTOL UAV navigation.

- Condition (22) means that the norm of $\dot{v}_{1,2}(t)$ is not null for all time and its direction is not constant for all time. As for condition (23), it is not satisfied if $\forall t: \frac{\dot{v}_{2}(t)}{\dot{v}_{1}(t)}=\frac{m_{2}}{m_{1}}$ which, a part from the case of constant horizontal linear velocity (i.e. $\dot{v}_{1} \equiv \dot{v}_{2} \equiv 0$ ), is also very particular. Note that even when both conditions (22) and (23) are violated, the condition of the third case, which does not rely on the horizontal linear accelerations, may still be satisfied. Moreover, all the 

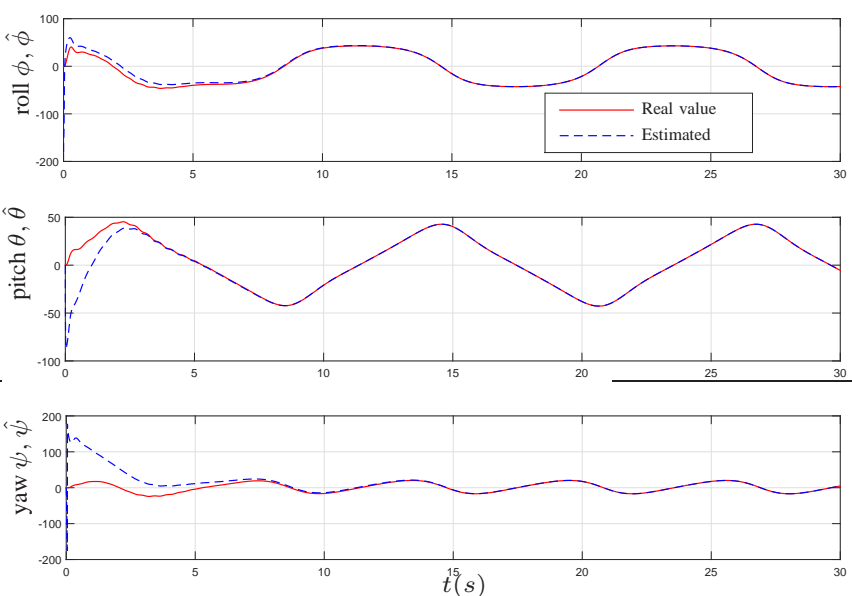

Fig. 1. Simulation 1: Estimated and real attitude represented by roll, pitch and yaw Euler angles $(\mathrm{deg})$ versus time $(s)$.
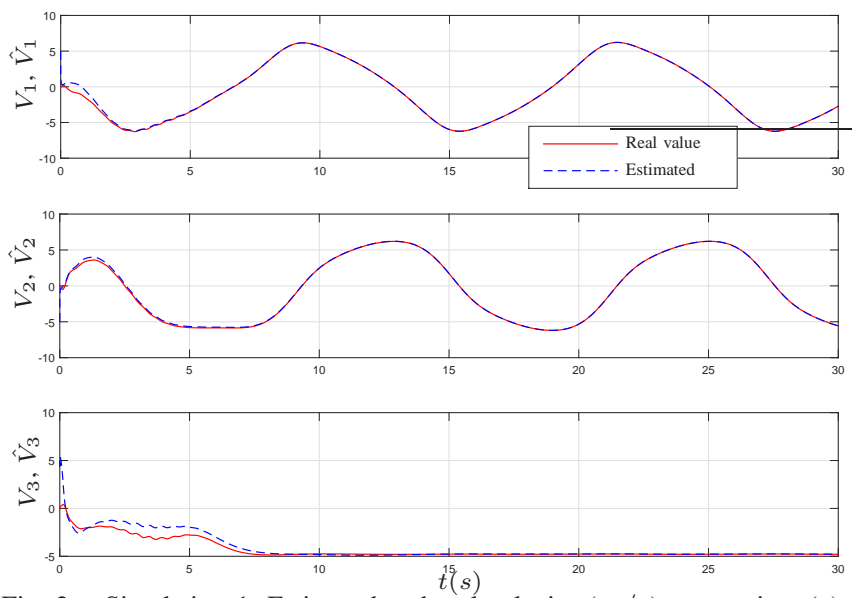

Fig. 2. Simulation 1: Estimated and real velocity $(\mathrm{m} / \mathrm{s})$ versus time $(s)$.

five cases (combined with condition (21)) only provide nonminimal sufficient conditions for the validity of condition (18) and their violation thus does not necessarily lead to the violation of (18).

\section{Simulation Results}

Simulations are conducted on a model of a vertical takeoff and landing (VTOL) aerial drone, also used in [6]. The vehicle is stabilized along a circular reference trajectory, with the linear velocity expressed in the inertial frame $\{\mathcal{I}\}$ given by $v_{r}=[-15 \alpha \sin (\alpha t) ; 15 \alpha \cos (\alpha t) ; 0](\mathrm{m} / \mathrm{s})$, with $\alpha=$ $2 / \sqrt{15}$. Due to aerodynamic forces the vehicle's orientation varies in large proportions. The normalized earth's magnetic field and the gravity constant are respectively equal to $m_{\mathcal{I}}=$ $[0.434 ;-0.0091 ; 0.9008]$ and $g=9.81\left(\mathrm{~m} / \mathrm{s}^{2}\right)$.

The proposed observer is tuned analogously to KalmanBucy filters where the matrices $S$ and $Q^{-1}$ are interpreted as covariance matrices of the additive noise on the system state and output respectively. The following parameters are chosen: $P(0)=\operatorname{diag}\left(2 I_{3}, 20 I_{3}\right), Q(t)=\operatorname{diag}\left(25 I_{3}, 100 I_{3}\right)$, $S(t)=\operatorname{diag}\left(0.01 I_{3}, I_{3}\right)$

Two simulations are reported next.

- Simulation 1: In this simulation, the observer is simulated in the ideal case (i.e. noise-free measurements) for a set of initial attitude and velocity estimates corresponding to the
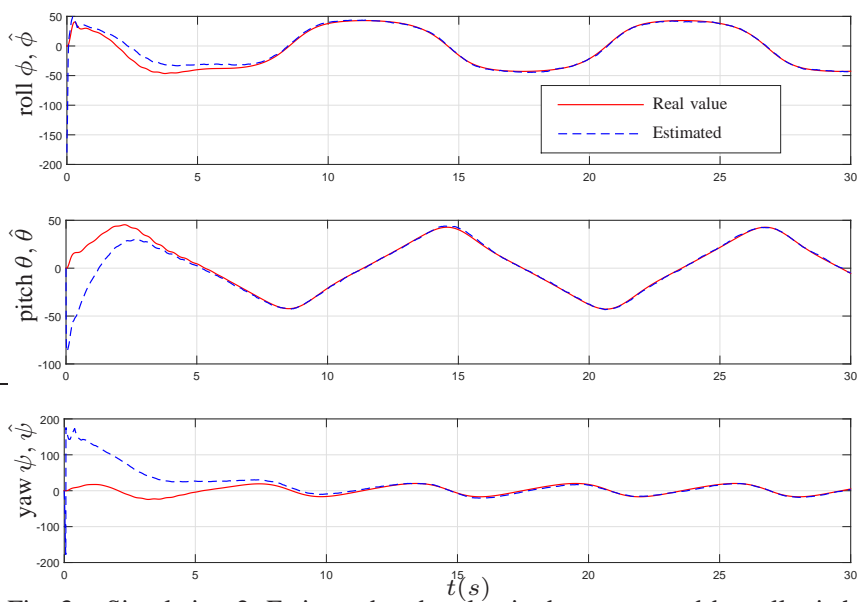

Fig. 3. Simulation 2: Estimated and real attitude represented by roll, pitch and yaw Euler angles (deg) versus time $(s)$
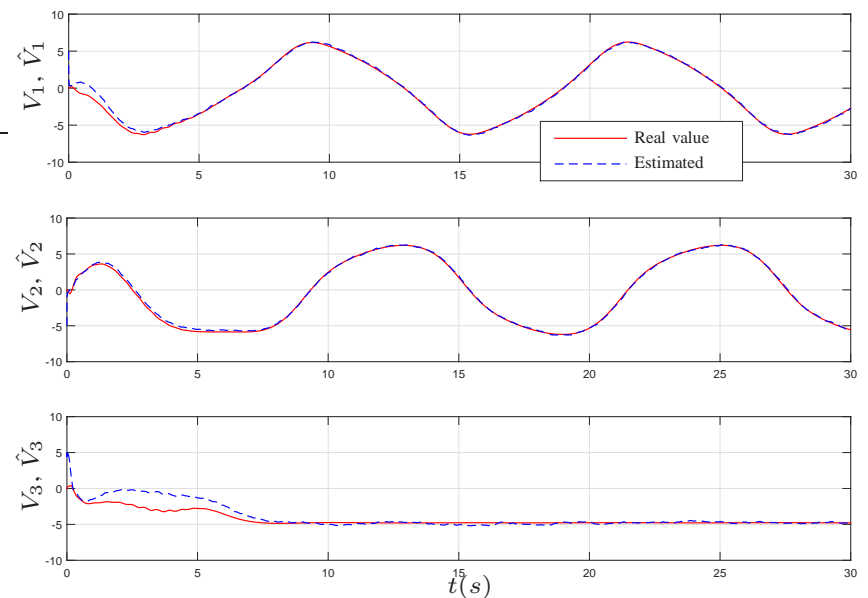

Fig. 4. Simulation 2: Estimated and real velocity $(\mathrm{m} / \mathrm{s})$ versus time $(\mathrm{s})$.

following initial estimation errors

$$
\left\{\begin{array}{l}
\tilde{v}(0)=[-5 ; 5 ;-5](\mathrm{m} / \mathrm{s}) \\
\tilde{q}(0)=\left[\cos \left(\frac{\pi}{2}\right) ; \sin \left(\frac{\pi}{2}\right) e_{1}\right]
\end{array}\right.
$$

This extreme case corresponds to an initial attitude error of $180(\mathrm{deg})$ in roll w.r.t. the true attitude. The time evolutions of the estimated and real attitudes, represented by Euler angles, along with the estimated and real velocity are shown in Figs. 1 and 2, respectively. The observer ensures the asymptotic convergence of the estimated variables to the real values despite the extremely large initial estimation errors, with quite good convergence rate.

- Simulation 2: This simulation is conducted with the same initial condition (24) as in Simulation 1. However, the measurements are now corrupted by Gaussian zero-mean additive noises with standard deviations reflecting the above choice of $Q\left(0.2 \mathrm{~m} / \mathrm{s}\right.$ for $v_{3}$ and $V_{1,2}$ and 0.1 for $\left.m_{\mathcal{B}}\right)$ and of $S\left(0.1 \mathrm{rad} / \mathrm{s}\right.$ for $\Omega$ and $1 \mathrm{~m} / \mathrm{s}^{2}$ for $\left.a_{\mathcal{B}}\right)$. Moreover, they are discretized with update frequencies of $20 \mathrm{~Hz}$, for the measurements of $V_{1}, V_{2}, v_{3}$ and $m_{\mathcal{B}}$, and of $50 \mathrm{~Hz}$, for the measurements of $\Omega$ and $a_{\mathcal{B}}$. The results reported in Figs. 3 and 4 indicate that important noises and low update frequencies of the measurements only marginally affect the overall performance of the observer. 


\section{CONCLUSIONS}

In this paper, a new problem of coupled velocity-aided attitude estimation has been addressed and a nonlinear observer has been proposed on the basis of a recent deterministic Riccati observer design framework. It is supported by comprehensive stability and observability analysis, and also by convincing simulation results.

Acknowledgement: This research was supported by the French Agence Nationale de la Recherche via the ASTRID SCAR (ANR-12-ASTR-0033) and ROBOTEX (ANR-10EQPX-44) projects.

\section{APPENDIX}

\section{A. Proof of Proposition 1}

Case 1: In this case $v \times e_{3} \equiv \dot{v} \times e_{3} \equiv 0$ and

$$
\begin{aligned}
& \frac{1}{\delta} \int_{t}^{t+\delta} X^{\top} D(s) X d s \\
& =\frac{1}{\delta} \int_{t}^{t+\delta}\left(\left|m_{\mathcal{I}} \times x\right|^{2}+\frac{\varepsilon_{r} g^{2}}{1+\varepsilon_{r}}\left|\left[R(s) e_{3}\right]_{\times}\left[e_{3}\right]_{\times} x\right|^{2}\right. \\
& +y_{1}^{2}+y_{2}^{2}+\left(\left(R^{\top}(s) e_{3}\right)^{\top} y\right)^{2}-\varepsilon_{r}\left|\left[e_{3}\right]_{\times}[\Omega(s)]_{\times} y\right|^{2} \\
& \left.+\left(\frac{g R^{\top}(s)\left[R(s) e_{3}\right]_{\times}\left[e_{3}\right]_{\times} x}{\sqrt{1+\varepsilon_{r}}}+\sqrt{1+\varepsilon_{r}}\left[e_{3}\right]_{\times}[\Omega(s)]_{\times} y\right)^{2}\right) d s \\
& \geq\left|m_{\mathcal{I}} \times x\right|^{2}+\frac{1}{\delta} \int_{t}^{t+\delta} \frac{\varepsilon_{r} g^{2}}{1+\varepsilon_{r}}\left|\left[R(s) e_{3}\right]_{\times}\left[e_{3}\right]_{\times} x\right|^{2} d s \\
& +y_{1}^{2}+y_{2}^{2}+\frac{1}{\delta} \int_{t}^{t+\delta}\left(\left(R^{\top}(s) e_{3}\right)^{\top} y\right)^{2} d s-\varepsilon_{r} \Omega_{\text {max }}^{2}|y|^{2}
\end{aligned}
$$

with $\varepsilon_{r}>0$ such that $\exists \mu_{y}^{r}>0$ :

$$
y_{1}^{2}+y_{2}^{2}+\frac{1}{\delta} \int_{t}^{t+\delta}\left(\left(R^{\top}(s) e_{3}\right)^{\top} y\right)^{2} d s \geq\left(\mu_{y}^{r}+\varepsilon_{r} \Omega_{\text {max }}^{2}\right)|y|^{2}
$$

A number $\varepsilon_{r}$ satisfying this inequality is calculated next. Defining $\gamma \triangleq R^{\top} e_{3} \in S^{2}$ one gets

$\left(\gamma^{\top} y\right)^{2}=\left(\gamma_{3} y_{3}\right)^{2}+2\left(\gamma_{1} y_{1}+\gamma_{2} y_{2}\right)\left(\gamma_{3} y_{3}\right)+\left(\gamma_{1} y_{1}+\gamma_{2} y_{2}\right)^{2}$ $\geq \frac{1}{3}\left(\gamma_{3} y_{3}\right)^{2}-\frac{1}{2}\left(\gamma_{1} y_{1}+\gamma_{2} y_{2}\right)^{2} \geq \frac{1}{3}\left(\gamma_{3} y_{3}\right)^{2}-\left(\gamma_{1} y_{1}\right)^{2}-\left(\gamma_{2} y_{2}\right)^{2}$ when using the following Young inequalities

$$
\begin{aligned}
& 2\left(\gamma_{1} y_{1}+\gamma_{2} y_{2}\right)\left(\gamma_{3} y_{3}\right) \geq-\frac{2}{3}\left(\gamma_{3} y_{3}\right)^{2}-\frac{3}{2}\left(\gamma_{1} y_{1}+\gamma_{2} y_{2}\right)^{2} \\
& \left(\gamma_{1} y_{1}+\gamma_{2} y_{2}\right)^{2} \leq 2\left(\left(\gamma_{1} y_{1}\right)^{2}+\left(\gamma_{2} y_{2}\right)^{2}\right)
\end{aligned}
$$

Since $\gamma_{3}=R_{3,3}$ one deduces from (21) that

$$
\begin{aligned}
y_{1}^{2} & +y_{2}^{2}+\frac{1}{\delta} \int_{t}^{t+\delta}\left(\left(R^{\top}(s) e_{3}\right)^{\top} y\right)^{2} d s-\varepsilon_{r} \Omega_{\text {max }}^{2}|y|^{2} \\
\geq & \frac{1}{\delta} \int_{t}^{t+\delta}\left(\left(1-\gamma_{1}^{2}(s)\right) y_{1}^{2}+\left(1-\gamma_{2}^{2}(s)\right) y_{2}^{2}+\frac{1}{3} \gamma_{3}^{2}(s) y_{3}^{2}\right) d s \\
& -\varepsilon_{r} \Omega_{\text {max }}^{2}|y|^{2} \\
\geq & \left(\frac{1}{3 \delta} \int_{t}^{t+\delta} \gamma_{3}^{2}(s) d s-\varepsilon_{r} \Omega_{\text {max }}^{2}\right)|y|^{2} \geq \mu_{y}^{r}|y|^{2}
\end{aligned}
$$

with $\mu_{y}^{r} \triangleq \rho / 3-\varepsilon_{r} \Omega_{\max }^{2}$. Therefore, any number $\varepsilon_{r}$ such that $0<\varepsilon_{r}<\rho /\left(3 \Omega_{\max }^{2}\right)$ ensures that $\mu_{y}^{r}$ in (27) is positive.

Let us now consider the term

$$
\left|m_{\mathcal{I}} \times x\right|^{2}+\frac{1}{\delta} \int_{t}^{t+\delta} \frac{\varepsilon_{r} g^{2}}{1+\varepsilon_{r}}\left|\left[R(s) e_{3}\right]_{\times}\left[e_{3}\right]_{\times} x\right|^{2} d s
$$

involved in the last inequality of (25). By simple computations one obtains

$$
\left[R e_{3}\right]_{\times}\left[e_{3}\right]_{\times} x=\left[\begin{array}{ccc}
-R_{3,3} & 0 & 0 \\
0 & -R_{3,3} & 0 \\
R_{1,3} & R_{2,3} & 0
\end{array}\right] x=\left[\begin{array}{c}
-R_{3,3} x_{1} \\
-R_{3,3} x_{2} \\
R_{1,3} x_{1}+R_{2,3} x_{2}
\end{array}\right]
$$

Thus, defining $\bar{\varepsilon}_{r} \triangleq \frac{\varepsilon_{r} g^{2}}{1+\varepsilon_{r}}$ and using (21) one deduces

$$
\begin{aligned}
& \left|m_{\mathcal{I}} \times x\right|^{2}+\frac{1}{\delta} \int_{t}^{t+\delta} \frac{\varepsilon_{r} g^{2}}{1+\varepsilon_{r}}\left|\left[R(s) e_{3}\right]_{\times}\left[e_{3}\right]_{\times} x\right|^{2} d s \\
& =\left(m_{3} x_{2}-m_{2} x_{3}\right)^{2}+\left(m_{3} x_{1}-m_{1} x_{3}\right)^{2}+\left(m_{2} x_{1}-m_{1} x_{2}\right)^{2} \\
& +\frac{1}{\delta} \int_{t}^{t+\delta}\left(\bar{\varepsilon}_{r} R_{3,3}^{2}(s)\left(x_{1}^{2}+x_{2}^{2}\right)+\bar{\varepsilon}_{r}\left(R_{1,3}(s) x_{1}+R_{2,3}(s) x_{2}\right)^{2}\right) d s \\
& \geq\left(m_{3} x_{2}-m_{2} x_{3}\right)^{2}+\left(m_{3} x_{1}-m_{1} x_{3}\right)^{2}+\bar{\varepsilon}_{r} \rho\left(x_{1}^{2}+x_{2}^{2}\right) \\
& =\left(\sqrt{m_{3}^{2}+\frac{\bar{\varepsilon}_{r} \rho}{2}} x_{2}-\frac{m_{2} m_{3}}{\sqrt{m_{3}^{2}+\frac{\bar{\varepsilon}_{r} \rho}{2}}} x_{3}\right)^{2} \\
& \quad+\left(\sqrt{m_{3}^{2}+\frac{\bar{\varepsilon}_{r} \rho}{2}} x_{1}-\frac{m_{1} m_{3}}{\sqrt{m_{3}^{2}+\frac{\bar{\varepsilon}_{r} \rho}{2}}} x_{3}\right)^{2} \\
& \quad+\frac{\bar{\varepsilon}_{r} \rho}{2}\left(x_{1}^{2}+x_{2}^{2}\right)+\frac{\left(m_{1}^{2}+m_{2}^{2}\right) \bar{\varepsilon}_{r} \rho}{2 m_{3}^{2}+\bar{\varepsilon}_{r} \rho} x_{3}^{2} \geq \mu_{x}^{r}|x|^{2}
\end{aligned}
$$

with $\mu_{x}^{r} \triangleq \min \left(\frac{\bar{\varepsilon}_{r} \rho}{2}, \frac{\left(m_{1}^{2}+m_{2}^{2}\right) \bar{\varepsilon}_{r} \rho}{2 m_{3}^{2}+\bar{\varepsilon}_{r} \rho}\right)$, which is positive since $m_{\mathcal{I}}$ and $e_{3}$ are non-collinear. From (25), (27), (28) one then deduces that $\frac{1}{\delta} \int_{t}^{t+\delta} X^{\top} D(s) X d s \geq \mu|X|^{2}$ with $\mu \triangleq$ $\min \left(\mu_{x}^{r}, \mu_{y}^{r}\right)>0$. This concludes the proof of the first case.

Case 2: The proof proceeds analogously to the proof of the first case. Since $\Omega(t) \equiv 0$, similarly to $(25)$ one deduces that the left-hand side of (20) satisfies

$$
\begin{aligned}
& \frac{1}{\delta} \int_{t}^{t+\delta} X^{\top} D(s) X d s \geq \\
& y_{1}^{2}+y_{2}^{2}+\frac{1}{\delta} \int_{t}^{t+\delta} \frac{\varepsilon_{t}}{1+\varepsilon_{t}}\left(\left(R^{\top}(s) e_{3}\right)^{\top} y\right)^{2} d s \\
& \quad+\left|m_{\mathcal{I}} \times x\right|^{2}+\frac{g^{2}}{\delta} \int_{t}^{t+\delta}\left|\left[R(s) e_{3}\right]_{\times}\left[e_{3}\right]_{\times} x\right|^{2} d s \\
& \quad-\varepsilon_{t} v_{1,2 \max }^{2}\left(x_{1}^{2}+x_{2}^{2}\right)
\end{aligned}
$$

with some $\varepsilon_{t}>0$. Relation (28) is now replaced by

$$
\begin{aligned}
\mid m_{\mathcal{I}} & \times\left. x\right|^{2}+\frac{g^{2}}{\delta} \int_{t}^{t+\delta}\left|\left[R(s) e_{3}\right]_{\times}\left[e_{3}\right]_{\times} x\right|^{2} d s-\varepsilon_{t} v_{1,2 \max }^{2}\left(x_{1}^{2}+x_{2}^{2}\right) \\
& \geq\left(\frac{g^{2} \rho}{2}-\varepsilon_{t} v_{1,2 \max }^{2}\right)\left(x_{1}^{2}+x_{2}^{2}\right)+\frac{\left(m_{1}^{2}+m_{2}^{2}\right) g^{2} \rho}{2 m_{3}^{2}+g^{2} \rho} x_{3}^{2} \\
& \geq \min \left(\frac{g^{2} \rho}{2}-\varepsilon_{t} v_{1,2 \max }^{2}, \frac{\left(m_{1}^{2}+m_{2}^{2}\right) g^{2} \rho}{2 m_{3}^{2}+g^{2} \rho}\right)|x|^{2} \\
& \geq \mu_{x}^{t}|x|^{2}
\end{aligned}
$$

with $\mu_{x}^{t} \triangleq \min \left(\frac{g^{2} \rho}{2}-\varepsilon_{t} v_{1,2 \max }^{2}, \frac{\left(m_{1}^{2}+m_{2}^{2}\right) g^{2} \rho}{2 m_{3}^{2}+g^{2} \rho}\right)$. This number is positive if $\varepsilon_{t}$ is chosen such that $0<\varepsilon_{t}<\frac{g^{2} \rho}{2 v_{1,2 \max }^{2}}$. Relation (27) is now replaced by

$$
\begin{aligned}
& y_{1}^{2}+y_{2}^{2}+\frac{1}{\delta} \int_{t}^{t+\delta} \frac{\varepsilon_{t}}{1+\varepsilon_{t}}\left(\left(R^{\top}(s) e_{3}\right)^{\top} y\right)^{2} d s \\
& \geq \frac{1}{\delta} \int_{t}^{t+\delta} \frac{\varepsilon_{t}}{1+\varepsilon_{t}}\left(y_{1}^{2}+y_{2}^{2}+\left(\gamma^{\top}(s) y\right)^{2}\right) d s \geq \mu_{y}^{t}|y|^{2}
\end{aligned}
$$

with $\mu_{y}^{t} \triangleq \frac{\varepsilon_{t} \rho}{3\left(1+\varepsilon_{t}\right)}$. From (29), (30), (31) one then (31)duces that $\frac{1}{\delta} \int_{t}^{t+\delta} X^{\top} D(s) X d s \geq \mu|X|^{2}$, with $\mu \triangleq$ $\min \left(\mu_{x}^{t}, \mu_{y}^{t}\right)>0$. This concludes the proof of this case.

Case 3: Using the same procedure as the one used to derive relations (25), (27), and (28) one deduces

$$
\begin{aligned}
& \frac{1}{\delta} \int_{t}^{t+\delta} X^{\top} D(s) X d s \\
& \quad \geq\left|m_{\mathcal{I}} \times x\right|^{2}+\frac{1}{\delta} \int_{t}^{t+\delta} \frac{\varepsilon_{2} g^{2}}{1+\varepsilon_{2}}\left|\left[R(s) e_{3}\right]_{\times}\left[e_{3}\right]_{\times} x\right|^{2} d s \\
& \quad-\varepsilon_{1} v_{1,2 \max }^{2}\left(x_{1}^{2}+x_{2}^{2}\right) \\
& \quad+y_{1}^{2}+y_{2}^{2}+\frac{1}{\delta} \int_{t}^{t+\delta} \frac{\varepsilon_{1}}{1+\varepsilon_{1}}\left(\left(R^{\top}(s) e_{3}\right)^{\top} y\right)^{2} d s-\left.\varepsilon_{2} \Omega_{\text {max }}^{2} y\right|^{2} \\
& \geq\left(\frac{\varepsilon_{2} g^{2} \rho}{2\left(1+\varepsilon_{2}\right)}-\varepsilon_{1} v_{1,2 \max }^{2}\right)\left(x_{1}^{2}+x_{2}^{2}\right)+\frac{\left(m_{1}^{2}+m_{2}^{2}\right) \bar{\varepsilon}_{2} \rho}{2 m_{3}^{2}+\bar{\varepsilon}_{2} \rho} x_{3}^{2} \\
& \quad+\left(\frac{\varepsilon_{1} \rho}{3\left(1+\varepsilon_{1}\right)}-\varepsilon_{2} \Omega_{\max }^{2}\right)|y|^{2}
\end{aligned}
$$


with $\bar{\varepsilon}_{2} \triangleq \frac{\varepsilon_{2} g^{2}}{1+\varepsilon_{2}}$ and $\varepsilon_{1}, \varepsilon_{2}>0$ chosen such that

$$
\left\{\begin{array}{l}
\frac{\varepsilon_{2}}{1+\varepsilon_{2}}>\alpha_{1} \varepsilon_{1}, \text { with } \alpha_{1} \triangleq \frac{2 v_{1,2 \max }^{2}}{g^{2} \rho} \\
\frac{\varepsilon_{1}}{1+\varepsilon_{1}}>\alpha_{2} \varepsilon_{2}, \text { with } \alpha_{2} \triangleq \frac{3 \Omega_{\max }^{2}}{\rho}
\end{array}\right.
$$

One then verifies that positive solutions of $\varepsilon_{1}$ and $\varepsilon_{2}$ to (32) exist if $\alpha_{1} \alpha_{2}<1$ or, equivalently, if $v_{1,2 \max } \Omega_{\max } \leq \frac{g \rho}{\sqrt{6}}$. From here (18) follows immediately.

Case 4: Using (22) one obtains

$$
\begin{aligned}
& \frac{1}{\delta} \int_{t}^{t+\delta}\left(\left(e_{3} \times \dot{v}(s)\right)^{\top} x\right)^{2} d s \\
& =\left[\begin{array}{c}
-x_{2} \\
x_{1}
\end{array}\right]^{\top}\left(\frac{1}{\delta} \int_{t}^{t+\delta} \dot{v}_{1,2}(s) \dot{v}_{1,2}^{\top}(s) d s\right)\left[\begin{array}{c}
-x_{2} \\
x_{1}
\end{array}\right] \\
& \geq \rho_{1}\left(x_{1}^{2}+x_{2}^{2}\right)
\end{aligned}
$$

Then, proceeding similarly to (25), (28), (31), and using (33) one deduces from the right-hand side of (20) that

$$
\begin{aligned}
& \frac{1}{\delta} \int_{t}^{t+\delta} X^{\top} D(s) X d s \\
= & \frac{1}{\delta} \int_{t}^{t+\delta}\left(\left|m_{\mathcal{I}} \times x\right|^{2}+\left(\left(e_{3} \times \dot{v}(s)\right)^{\top} x\right)^{2}-\varepsilon_{1}\left(\left(e_{3} \times v(s)\right)^{\top} x\right)^{2}\right. \\
& +y_{1}^{2}+y_{2}^{2}+\frac{\varepsilon_{1}}{1+\varepsilon_{1}}\left(\left(R^{\top}(s) e_{3}\right)^{\top} y\right)^{2} \\
& +\left(\sqrt{1+\varepsilon_{1}}\left(v(s) \times e_{3}\right)^{\top} x+\frac{\left(R^{\top}(s) e_{3}\right)^{\top} y}{\sqrt{1+\varepsilon_{1}}}\right)^{2} \\
& \left.+\left|g R^{\top}(s)\left[R(s) e_{3}\right]_{\times}\left[e_{3}\right]_{\times} x+\left[e_{3}\right]_{\times}[\Omega(s)]_{\times} y\right|^{2}\right) d s \\
\geq & \left|m_{\mathcal{I}} \times x\right|^{2}+\bar{\rho}_{1}\left(x_{1}^{2}+x_{2}^{2}\right)+\frac{\varepsilon_{1} \rho}{3\left(1+\varepsilon_{1}\right)}|y|^{2} \\
\geq & \frac{\bar{\rho}_{1}}{2}\left(x_{1}^{2}+x_{2}^{2}\right)+\frac{\left(m_{1}^{2}+m_{2}^{2}\right) \bar{\rho}_{1} x_{3}^{2}+\frac{\varepsilon_{1} \rho}{2 m_{3}^{2}+\bar{\rho}_{1}}|y|^{2}}{3\left(1+\varepsilon_{1}\right)}
\end{aligned}
$$

with $0<\varepsilon_{1}<\rho_{1} / v_{1,2 \max }^{2}$ and $\bar{\rho}_{1} \triangleq \rho_{1}-\varepsilon_{1} v_{1,2 \max }^{2}>0$. From here one easily deduces (18).

Case 5: From the first equality of (34) and using (21), one deduces

$$
\begin{aligned}
& \frac{1}{\delta} \int_{t}^{t+\delta} X^{\top} D(s) X d s \\
& \geq\left(m_{2} x_{1}-m_{1} x_{2}\right)^{2}+\frac{1}{\delta} \int_{t}^{t+\delta}\left(-\dot{v}_{2}(s) x_{1}+\dot{v}_{1}(s) x_{2}\right)^{2} d s \\
& \quad+\left(m_{3} x_{2}-m_{2} x_{3}\right)^{2}+\left(m_{3} x_{1}-m_{1} x_{3}\right)^{2} \\
& \quad-\varepsilon_{1} v_{1,2 \max }^{2}\left(x_{1}^{2}+x_{2}^{2}\right)+\frac{\varepsilon_{1} \rho}{3\left(1+\varepsilon_{1}\right)}|y|^{2}
\end{aligned}
$$

with $\varepsilon_{1}>0$ specified hereafter. Defining

$$
\left[\begin{array}{l}
\bar{x}_{1} \\
\bar{x}_{2}
\end{array}\right] \triangleq\left[\begin{array}{cc}
\frac{m_{1}}{\sqrt{m_{1}^{2}+m_{2}^{2}}} & \frac{m_{2}}{\sqrt{m_{1}^{2}+m_{2}^{2}}} \\
-\frac{m_{2}}{\sqrt{m_{1}^{2}+m_{2}^{2}}} & \frac{m_{1}}{\sqrt{m_{1}^{2}+m_{2}^{2}}}
\end{array}\right]\left[\begin{array}{l}
x_{1} \\
x_{2}
\end{array}\right]
$$

and using (23) one deduces

$$
\begin{aligned}
& \left.\frac{1}{\delta} \int_{t}^{t+\dot{\xi}}-\dot{v}_{2}(s) x_{1}+\dot{v}_{1}(s) x_{2}\right)^{2} d s \\
& =\frac{1}{\left(m_{1}^{2}+m_{2}^{2}\right) \delta} \int_{t}^{t+\delta}\left(\left(-m_{1} \dot{v}_{2}(s)+m_{2} \dot{v}_{1}(s)\right) \bar{x}_{1}\right. \\
& \left.+\left(m_{1} \dot{v}_{1}(s)+m_{2} \dot{v}_{2}(s)\right) \bar{x}_{2}\right)^{2} d s \\
& =\frac{\varepsilon_{2}}{\left(1+\varepsilon_{2}\right)\left(m_{1}^{2}+m_{2}^{2}\right)}\left(\frac{1}{\delta} \int_{t}^{t+\delta}\left(-m_{1} \dot{v}_{2}(s)+m_{2} \dot{v}_{1}(s)\right)^{2} d s\right) \bar{x}_{1}^{2} \\
& -\frac{\varepsilon_{2}}{m_{1}^{2}+m_{2}^{2}}\left(\frac{1}{\delta} \int_{t}^{t+\delta}\left(m_{1} \dot{v}_{1}(s)+m_{2} \dot{v}_{2}(s)\right)^{2} d s\right) \bar{x}_{2}^{2} \\
& +\frac{1}{\left(m_{1}^{2}+m_{2}^{2}\right) \delta} \int_{t}^{t+\delta}\left(\frac{-m_{1} \dot{v}_{2}(s)+m_{2} \dot{v}_{1}(s)}{\sqrt{1+\varepsilon_{2}}} \bar{x}_{1}\right. \\
& \left.+\sqrt{1+\varepsilon_{2}}\left(m_{1} \dot{v}_{1}(s)+m_{2} \dot{v}_{2}(s)\right) \bar{x}_{2}\right)^{2} d s \\
& \geq \frac{\varepsilon_{2} \rho_{2}}{\left(1+\varepsilon_{2}\right)\left(m_{1}^{2}+m_{2}^{2}\right)} \bar{x}_{1}^{2}-\varepsilon_{2} \dot{v}_{1,2 \max }^{2} \bar{x}_{2}^{2}
\end{aligned}
$$

Choosing any positive number $\varepsilon_{2}<\frac{m_{1}^{2}+m_{2}^{2}}{\dot{v}_{1,2 \max }^{2}}$ and defining $\bar{\varepsilon}_{2} \triangleq \min \left(\frac{\varepsilon_{2} \rho_{2}}{\left(1+\varepsilon_{2}\right)\left(m_{1}^{2}+m_{2}^{2}\right)}, m_{1}^{2}+m_{2}^{2}-\varepsilon_{2} \dot{v}_{1,2 \max }^{2}\right)>0$, one deduces from (35), (36) and the relations

$$
\left(m_{2} x_{1}-m_{1} x_{2}\right)^{2}=\left(m_{1}^{2}+m_{2}^{2}\right) \bar{x}_{2}^{2}, \quad \bar{x}_{1}^{2}+\bar{x}_{2}^{2}=x_{1}^{2}+x_{2}^{2}
$$

that

$$
\begin{aligned}
& \frac{1}{\delta} \int_{t}^{t+\delta} X^{\top} D(s) X d s \\
\geq & \bar{\varepsilon}_{2}\left(x_{1}^{2}+x_{2}^{2}\right)+\left(m_{3} x_{2}-m_{2} x_{3}\right)^{2}+\left(m_{3} x_{1}-m_{1} x_{3}\right)^{2} \\
& -\varepsilon_{1} v_{1,2 \max }^{2}\left(x_{1}^{2}+x_{2}^{2}\right)+\frac{\varepsilon_{1} \rho}{3\left(1+\varepsilon_{1}\right)}|y|^{2} \\
\geq & \left(\frac{\bar{\varepsilon}_{2}}{2}-\varepsilon_{1} v_{1,2 \max }^{2}\right)\left(x_{1}^{2}+x_{2}^{2}\right)+\frac{\left(m_{1}^{2}+m_{2}^{2}\right) \bar{\varepsilon}_{2}}{2 m_{3}^{2}+\bar{\varepsilon}_{2}} x_{3}^{2} \\
& +\frac{\varepsilon_{1} \rho}{3\left(1+\varepsilon_{1}\right)}|y|^{2}
\end{aligned}
$$

where the last inequality is obtained by proceeding analogously to (28). From here by choosing any positive number $\varepsilon_{1}<\frac{\bar{\varepsilon}_{2}}{2 v_{1,2 \max }^{2}}$, (18) then directly follows.

\section{REFERENCES}

[1] G. Allibert, R. Mahony, and M. Bangura. Velocity aided attitude estimation for aerial robotic vehicles using latent rotation scaling. In IEEE Int. Conf. on Robotics and Automation, pages 1538-1543, 2016.

[2] P. Batista, C. Silvestre, and P. Oliveira. Sensor-based globally asymptotically stable filters for attitude estimation: Analysis, design, and performance evaluation. IEEE Transactions on Automatic Control, 57(8):2095-2100, 2012.

[3] S. Bonnabel, P. Martin, and P. Rouchon. Symmetry-preserving observers. IEEE Trans. on Autom. Control, 53(11):2514-2526, 2008.

[4] H. F. Grip, T. I. Fossen, T. A. Johansen, and A. Saberi. A nonlinear observer for integration of GNSS and IMU measurements with gyro bias estimation. In American Cont. Conf., pages 4607-4612, 2012.

[5] T. Hamel and C. Samson. Riccati observers for the non-stationary PnP problem. 2017. IEEE Trans. on Automatic Control.

[6] M.-D. Hua. Attitude estimation for accelerated vehicles using GPS/INS measurements. Control Eng. Pract., 18(7):723-732, 2010.

[7] M.-D. Hua, G. Ducard, T. Hamel, R. Mahony, and K. Rudin. Implementation of a nonlinear attitude estimator for aerial robotic vehicles. IEEE Trans. on Control Syst. Technol., 22(1):201-213, 2014.

[8] M.-D. Hua, P. Martin, and T. Hamel. Stability analysis of velocityaided attitude observers for accelerated vehicles. Automatica, 63:1115, 2016.

[9] A. Khosravian, J. Trumpf, R. Mahony, and T. Hamel. State estimation for invariant systems on lie groups with delayed output measurements. Automatica, 68:254-265, 2016

[10] R. Mahony, T. Hamel, and J.-M. Pflimlin. Nonlinear complementary filters on the special orthogonal group. IEEE Trans. on Autom. Control, 53(5):1203-1218, 2008.

[11] R. Mahony, V. Kumar, and P. Corke. Multirotor aerial vehicles: Modeling, estimation, and control of quadrotor. IEEE Robotics \& Automation Magazine, 19(3):20-32, 2012.

[12] P. Martin and E. Salaün. An invariant observer for Earth-VelocityAided attitude heading reference systems. In IFAC World Congr. pages 9857-9864, 2008.

[13] P. Martin and E. Salaun. Design and implementation of a low-cost observer-based attitude and heading reference system. Control Eng. Pract., 18(7):712-722, 2010.

[14] P. Martin and E. Salaün. The true role of accelerometer feedback in quadrotor control. In IEEE Int. Conf. on Robotics and Automation, pages $1623-1629,2010$.

[15] A. Roberts and A. Tayebi. On the attitude estimation of accelerating rigid-bodies using GPS and IMU measurements. In IEEE Conf. on Dec. and Cont., pages 8088-8093, 2011.

[16] G. G. Scandaroli. Visuo-inertial data fusion for pose estimation and self-calibration. PhD thesis, Université Nice Sophia Antipolis, 2013.

[17] G. Troni and L. L. Whitcomb. Preliminary experimental evaluation of a Doppler-aided attitude estimator for improved Doppler navigation of underwater vehicles. In IEEE Int. Conf. on Robotics and Automation, pages 4134-4140, 2013. 